\title{
Estrategias para disminuir las causas que afectan el rendimiento académico en la tropa del servicio militar voluntario, en Suyo - Perú
}

\section{Strategies to reduce the causes that affect academic performance in the voluntary military service troop, in Suyo - Peru}

DOI: $10.46932 / \operatorname{sjdv2n5-148}$

Received in: Oct 1st, 2021

Accepted in: Dec 30th, 2021

\section{Luis Alberto Saavedra Saldaña \\ Universidad César Vallejo - Piura, Perú. \\ Tte. Crl. del Ejército del Perú.}

Egresado como oficial de la Escuela Militar de Chorrillos en el año 1999, licenciado en Ciencias

Militares con mención en Administración-especialización de Caballería, graduado como Maestro de Equitación del Ejército,Maestro en Ciencias Militares con mención en Planeamiento Estratégico y Toma de Decisiones.

Villa militar Lomas de Teodomiro Block C 202.

E-mail: saavedral335@gmail.com

\section{Mario Napoleón Briones Mendoza}

Universidad César Vallejo - Piura, Perú.

Doctor en Educación.

Docente de la Escuela de Post Grado de la Universidad César Vallejo.

Director de la I.E. "Libertadores de América"- La Unión Piura- Perú.

El Indio Mz. c5 lote 10 Castilla - Piura - Perú.

E-mail: saavedral@gmail.com

\begin{abstract}
RESUMEN
Las causas que afectan en el rendimiento académico al personal militar son múltiples, siendo importantes poner la atención en factores como personales, familia, docente, escuela, regimiento y los afectivos. El propósito fue diseñar una propuesta de estrategias para disminuir las causas que afectan el rendimiento académico. La metodología fue no experimental, transaccional proyectiva, la muestra fue de 128 soldados del servicio militar voluntario del Regimiento Gran Mariscal Necochea $N^{\circ} 7$ Suyo, se utilizó un cuestionario de 46 ítems, validado por expertos cuya confiabilidad fue de Alfa = ,894. Resultados: En los factores asociados al estudiante, existe escasa responsabilidad y no escasos hábitos de estudio. Factor familia, bajo apoyo y medios económicos bajos. Factor docente, baja asistencia y responsabilidad en las labores académicas, trato y enseñanza deficiente. Factor escuela; no se cuenta con los medios y materiales suficientes. Facto motivación y afectivo, baja motivación, dificultades en las relaciones interpersonales. Factor regimiento escasas condiciones para el estudio y ambientes no apropiados. Conclusión: Dada la importancia de los factores mencionados y que están afectando el rendimiento académico es necesario hacer una propuesta de estrategias metodológicas con fundamento científico que permitan reducir esta problemática encontrada.
\end{abstract}

Palabras clave: Familia, rendimiento académico, motivación, regimiento, factores estudiantes y familia. 


\begin{abstract}
The causes that affect the academic performance of military personnel are multiple, being important to pay attention to factors such as personal, family, teacher, school, regiment and affective. The purpose was to design a proposal of strategies to reduce the causes that affect academic performance. The methodology was non-experimental, projective transactional, the sample was 128 soldiers from the voluntary military service of the Regiment Gran Mariscal Necochea No. 7 Suyo, a questionnaire of 46 items was used, validated by experts whose reliability was Alpha $=.894$. Results: In the factors associated with the student, there is little responsibility and not few study habits. Family factor, low support and low economic means. Teaching factor, low attendance and responsibility in academic work, poor treatment and teaching. School factor; there are not enough means and materials. Motivational and affective factor, low motivation, difficulties in interpersonal relationships. Regiment factor, scarce study conditions and inappropriate environments. Conclusion: Given the importance of the aforementioned factors and that are affecting academic performance, it is necessary to make a proposal of methodological strategies with scientific basis that allow reducing this problem found.
\end{abstract}

Keywords: Family, academic performance, motivation, regiment, student and family factors

\title{
1 INTRODUCCIÓN
}

La actual situación que vive el mundo debido a la pandemia, ha develado las grandes deficiencias del sector educativo, Lamas (2015), sostiene que el análisis del rendimiento académico de los estudiantes es, por su trascendencia y complejidad, uno de los aspectos de mayor discusión en la indagación educativa, y se le ha brindado especial cuidado en las últimas décadas, en este sentido Rodríguez y Madrigal (2016) señalan que al rendimiento académico se le considera un indicador de eficacia y calidad educativa, sin embargo, también se le concibe como un problema multifactorial en el que participan diferentes variables, como: la práctica docente, tipo de asignatura, entorno familiar, apoyo institucional, motivación, entre otras.

En el Perú, el Ministerio de Educación (MINEDU, 2019) reporta que los logros alcanzados por los estudiantes, específicamente en la lectura de diversos tipos de textos en su lengua materna, a nivel nacional sólo el $14.5 \%$ de estudiantes evaluados alcanza el nivel satisfactorio en esta competencia, evidenciando dificultades para la comprensión de los aprendizajes. Los estudios revelan además que existen factores que intervienen en el rendimiento académico, en este sentido Grade (2018) revela como factores a los aspectos como las características de los estudiantes, sus familias, del docente, del aula y escuela, resaltando que los estudiantes que provienen de hogares con mayores precariedades sociales, económicas y materiales son aquellos que obtienen resultados poco favorables.

Lo mismo señala Sánchez (2017) considera que los factores personales, sociales e institucionales son los intervienen en el logro de los aprendizajes, de igual forma López (2017) manifiesta que las variables que más inciden en el abandono escolar de los estudiantes son: Condiciones económicas, ambiente familiar y estado académico. Podemos evidenciar que factores como familia, situación 
económica, aspectos sociales y motivaciones juegan un rol importante en los aprendizajes de los estudiantes.

Fajardo y Maestre (2017) demuestran que la formación académica de los padres de familia el tipo de ocupación el apoyo hacia las tares y la concepción que consideran los alumnos que tienen sus padres sobre su apreciación como estudiantes tienen una importante influencia sobre el rendimiento académico, esto muestra coincidencia con lo que manifiesta Garijo (2016), el autor concluye que el esfuerzo, motivación del logro, el autoconcepto social y académico, habilidades sociales, familia y expectativas de los estudiantes, influyen en sus logros académicos. Por su parte Velasco (2016) señala que los factores que más se relacionan con rendimiento académico exitoso es el contexto, la escuela, aula y alumnado.

Es necesario que a partir de la problemática se puedan generar estrategias que permitan reducir la problemática del rendimiento académico. En ese sentido una serie de investigaciones se han centrado es realizar propuestas que pueden contribuir a reducir esta problemática, es así que Carrizales (2017) encuentra deficiencias en el rendimiento académico de los estudiantes, sin embargo, al aplicar estrategias demuestra mejoras significativas, asimismo. Buelvas (2018) destaca que el bueno uso de estrategias de aprendizaje genera buenos aprendizajes tanto en hombres como en mujeres. Programas con estrategias didácticas cognitivas también se vienen usando como medio para reducir las dificultades en los aprendizajes (Pedraza, 2016) y otras estrategias como la motivación (Méndez, 2016).

Los estudios arriba presentados, denotan la importancia de desarrollar una propuesta de estrategias que contribuya en la reducción de los factores personales, familiares, docente, escuela, motivación afecto y regimiento los cuales intervienen en el rendimiento académico en tiempos actuales. De acuerdo con lo mencionado el objetivo general del presente estudio es diseñar una propuesta de estrategias para disminuir las causas que afectan el rendimiento académico en la tropa del servicio militar voluntario, tomando como factores los aspectos personales, familiares, docente, escuela, la motivación y el regimiento.

El artículo se divide en secciones, la parte dos centrado en la revisión de los principales fundamentos teóricos que explican las causas que afectan el rendimiento académico, la parte tres se describen los métodos y materiales, la parte cuatro se presentan los resultados asociados a los objetivos de investigación acompañados de la interpretación, la quinta parte del documento se presenta la discusión, la sexta parte se estructuran las conclusiones y la última parte se presentan las referencias usadas en esta documento.

El rendimiento académico, en esta misma línea Adell (2006), agrega que es el conocimiento manifestado en una asignatura de acuerdo con le edad y nivel académico que le concierne al estudiante. Salas (2004) sostiene que, cuando al estudiante le afectan situaciones de las cuales le es bastante difícil 
salir, generalmente sufre las consecuencias del fracaso escolar, entendiéndose a este como el hecho de no lograr las competencias básicas para desenvolverse de forma independiente y autónoma en la sociedad.

Dentro de los modelos que intentan explicar los factores que pueden intervenir en el fracaso escolar, esta Gonzales (2003), sostiene que luego de analizar diversos estudios cuyos propósitos fueron determinar los factores que influyen sobre el rendimiento de los estudiantes, se pueden agrupar dichas causas en tres clases primordiales de factores escolares, analizados en la literatura de las instituciones educativas eficaces (Creemers y Scheerens, 1989; Gómez, 1982; Good y Brophy, 1986; Purkey y Smith, 1985). Parecen tener más incidencia en el rendimiento académico aspectos de naturaleza procesual y funcional (clima escolar) en relación a los aspectos estructurales (recursos materiales). Factores Personales. Con estos factores se dio inicio al estudio de las causas que influían en el rendimiento. Se enfatiza como las más significativas la inteligencia (la cual muestra correlación moderada y bastante variable con el rendimiento - Álvaro, 1990; Brengelmann, 1975a; Rodríguez, 1982a-), también, la motivación (la cual se relaciona bastante con las facultades causales y se asocia de forma positiva con el rendimiento -INCIE, 1976; Marsh, 1984; Pelechano, 1989; Weiner, 2000-), finalmente, el autoconcepto (con relación alta y significativamente con el rendimiento - Marsh, 1984, Song y Hattie, 1984) y los factores Contextuales. Lo encontrado en este tipo de estudios parece ser más discordante. Por un lado, el nivel sociocultural parece incidir de forma determinante en el rendimiento (Carabaña, 1979) mientras que, por otro lado, no se evidencian correlaciones y, en todo caso, cuando se hallan, estas son muy débiles (Pelechano, 1989).

Otro de los modelos más actuales y que amplia al conjunto de factores, propone factores como los sociales, familia, sistema educativo, alumno y aula (Marchessí, 2003). Por otro lado, desde la percepción del docente existen causas promueven el fracaso escolar, Barreiro (2001) detalla que los docentes aluden al fracaso escolar a la falta de hábitos de estudio, falta de motivación, falta de atención y concentración, escasa planificación por parte del estudiante, poca importancia al estudio, falta de apoyo de la familia, clima familiar inadecuado, priorización de los estudiantes en temas no académicos y desinterés.

De acuerdo a Barreiro (2001), existen causas que promueven el fracaso escolar desde la óptica de los docentes, las cuales son: La falta de hábitos que promuevan el estudio y los métodos de trabajo de desarrollo cognoscitivo, la ausencia de estimulación e interés para formarse, la falta de atención y concentración durante el desarrollo de las sesiones de aprendizajes, las limitaciones que se presentan en la comunicación por parte del estudiante, a nivel oral como escrito, la falta de planificación y/o priorización de tiempo dedicado al estudio, la excesiva carga de estudiantes que rebasa la cantidad limite por aula, la poca importancia que el estudiante brinda al desarrollo de aprendizajes, los problemas personales que aquejan al estudiante, la falta de apoyo escolar por parte de los padres de familia, clima familiar inadecuado, el estudiante prioriza mayor tiempo en actividades de ocio como: tv, reuniones con 
amigos, deportes, la realización de estudios impuestos de manera obligatoria por los familiares, el estudiante no cuenta en el hogar con las condiciones básicas para desarrollar sus tareas escolares y la poca comunicación de los padres de familia con sus hijos, especialmente en temas relacionados a problemas personales.

La propuesta de estrategias para incrementar el rendimiento académico de los soldados del Regimiento, se ha elaborado teniendo como fundamento teórico a diversos autores, y bajo las orientaciones e informes emitidos por el Ministerio de Educación, la Defensoría del Pueblo y el Congreso de la República del Perú. Los autores como Orellana (2020), destacan que a partir de estas estrategias se le debe enseñar a los estudiantes a asumir la responsabilidad de su propio aprendizaje resulta muy significativo y en relación a ello, propone pautas para desarrollar la responsabilidad en los estudiantes. También se ha tenido en cuenta a Cartagena (2008), destaca el desarrollo de hábitos de estudio acompañado de una buena planificación del tiempo, según como lo indica Calero (2005), que, en relación a la planificación del tiempo, señala que esta acción permite utilizar el tiempo de una manera eficaz y eficiente, obteniéndose resultados provechosos y reteniendo un máximo de conocimientos. En relación al ambiente Iglesias (2008) manifiesta que este debe ser lo más cálido posible para lograr aprendizajes significativos.

Sobre el apoyo, motivación y valoración de la familia en la educación de sus hijos, Álvarez (2006), sostiene que, cuanto mayor es el nivel de implicación de las familias en la educación de los hijos, mayor es el interés y la motivación que estos manifiestan en sus estudios.

El Ministerio de Educación (2016), brinda a través del Currículo Nacional de Educación Básica, las orientaciones metodológicas que deben ser tomadas en cuenta por los docentes en la planificación, ejecución y evaluación de los procesos de enseñanza y aprendizaje, con la finalidad de desarrollar aprendizajes significativos en los estudiantes. Señala que es preciso se tengan en cuenta los siguientes procesos metodológicos: Partir de situaciones significativas, generar interés y disposición como condición para el aprendizaje, aprender haciendo, el desarrollo de las competencias se coloca en la perspectiva de la denominada «enseñanza situada», para la cual aprender y hacer son procesos indesligables, es decir, la actividad y el contexto son claves para el aprendizaje. Partir de los saberes previos, construir el nuevo conocimiento, aprender del error o el error constructivo. El error suele ser considerado solo como síntoma de que el proceso de aprendizaje no va bien y que el estudiante presenta deficiencias. Generar el conflicto cognitivo, mediar el progreso de los estudiantes de un nivel de aprendizaje a otro superior, promover el trabajo cooperativo y promover el pensamiento complejo. El objetivo de la propuesta es diseñar estrategias para disminuir las causas que afectan el rendimiento académico de los soldados del Regimiento Gran Mariscal Necochea N ${ }^{\circ} 7$ Suyo, en el año 2021. 


\section{METODOLOGÍA}

En cuanto a la metodología el estudio fue descriptivo, no experimental, transaccional, propositivo, con una muestra de 120 estudiantes soldados del servicio militar voluntario del Regimiento Gran Mariscal Necochea $\mathrm{N}^{\circ} 7$ Suyo, se aplicó un cuestionario de 46 ítems construido para la presente investigación, validado por expertos y con procesos de confiabilidad fue de Alfa = ,894. El cuestionario consta de 46 ítems: Factores en el estudiante (9), Factores en la familia (8), Factores en el docente y práctica en el aula (11), Factores de la escuela (5), Factores motivacionales y afectivos (8) y Factores en el regimiento (5), se contó con el consentimiento informado de todos los involucrados en el estudio, los datos han sido analizados con tablas descriptivas tomando en cuenta cada una de las dimensiones de la variable.

\section{RESULTADOS}

Después de analizar los datos recogidos a partir de la encuesta aplicada a la unidad de análisis se presentan los resultados según los objetivos planteados, utilizando tablas de frecuencia, las primeras tablas dan cuenta de la problemática existente en cuanto a las causas que afectan el rendimiento académico y se presenta además un resumen de la temática que podría tener la propuesta para mitigar el problema encontrado.

Tabla 1 Factores personales que afectan el rendimiento académico de los estudiantes de la tropa.

\begin{tabular}{|c|c|c|c|c|c|c|c|c|}
\hline & \multicolumn{2}{|c|}{ Nunca } & \multicolumn{2}{|c|}{ Casi nunca } & \multicolumn{2}{|c|}{ Casi siempre } & \multicolumn{2}{|c|}{ Siempre } \\
\hline & $\mathrm{fi}$ & $\%$ & fi & $\%$ & $\mathrm{fi}$ & $\%$ & fi & $\%$ \\
\hline 1. Asistes con normalidad a las clases & 33 & $27,5 \%$ & 67 & $55,8 \%$ & 19 & $15,8 \%$ & 1 & $0,8 \%$ \\
\hline $\begin{array}{l}\text { 2. Tienes problemas para asistir a } \\
\text { clases }\end{array}$ & 30 & $25,0 \%$ & 71 & $59,2 \%$ & 19 & $15,8 \%$ & 0 & $0,0 \%$ \\
\hline $\begin{array}{l}\text { 3. Planificas tu tiempo para } \\
\text { organizarte en tus estudios }\end{array}$ & 15 & $12,5 \%$ & 83 & $69,2 \%$ & 22 & $18,3 \%$ & 0 & $0,0 \%$ \\
\hline $\begin{array}{l}\text { 4. Tienes un horario donde registras } \\
\text { tus actividades académicas }\end{array}$ & 18 & $15,0 \%$ & 83 & $69,2 \%$ & 19 & $15,8 \%$ & 0 & $0,0 \%$ \\
\hline $\begin{array}{l}\text { 5. Utilizas algún método de estudio } \\
\text { para adquirir conocimientos }\end{array}$ & 13 & $10,8 \%$ & 95 & $79,2 \%$ & 12 & $10,0 \%$ & 0 & $0,0 \%$ \\
\hline $\begin{array}{l}\text { 6. Tienes tiempo suficiente para } \\
\text { realizar las tareas escolares }\end{array}$ & 25 & $20,8 \%$ & 67 & $55,8 \%$ & 28 & $23,3 \%$ & 0 & $0,0 \%$ \\
\hline $\begin{array}{l}\text { 7. Tienes un ambiente adecuado para } \\
\text { realizar tus tareas }\end{array}$ & 22 & $18,3 \%$ & 84 & $70,0 \%$ & 14 & $11,7 \%$ & 0 & $0,0 \%$ \\
\hline $\begin{array}{l}\text { 8. Utilizas libros que te ayudan a } \\
\text { desarrollar tus tareas }\end{array}$ & 21 & $17,5 \%$ & 84 & $70,0 \%$ & 15 & $12,5 \%$ & 0 & $0,0 \%$ \\
\hline $\begin{array}{l}\text { 9. Utilizas algún dispositivo } \\
\text { electrónico para tus estudios (Celular, } \\
\text { Tablet, laptop, computadora) }\end{array}$ & 17 & $14,2 \%$ & 91 & $75,8 \%$ & 12 & $10,0 \%$ & 0 & $0,0 \%$ \\
\hline
\end{tabular}

Nota: Instrumento de evaluación

En la tabla 1, se puede observar los resultados sobre las características personales del personal de tropa referido a las causas que afectan el rendimiento académico, se observan un escasa responsabilidad $(59,2 \%)$ con sus labores académicas, no hay una buena planificación del tiempo, el 69,2\% no tiene buenos 
hábitos de estudios, además el 55,8\% señala que no tiene tiempo para estudiar, agregado a esto el 70\% no cuenta con un ambiente adecuado para realizar las tareas, y carecen de dispositivos para hacer sus trabajos $(75,8 \%)$. En términos generales existen dificultades personales en los estudiantes evaluados que se convierten un factor que afecta el rendimiento académico.

Tabla 2. Factores familiares que afectan el rendimiento académico de los estudiantes de la tropa.

\begin{tabular}{|c|c|c|c|c|c|c|c|c|}
\hline & \multicolumn{2}{|c|}{ Nunca } & \multicolumn{2}{|c|}{ Casi nunca } & \multicolumn{2}{|c|}{ Casi siempre } & \multicolumn{2}{|c|}{ Siempre } \\
\hline & fi & $\%$ & fi & $\%$ & fi & $\%$ & fi & $\%$ \\
\hline $\begin{array}{l}\text { 1. Tu familia te brinda apoyo para que } \\
\text { puedas realizar tus tareas }\end{array}$ & 12 & $10,0 \%$ & 59 & $49,2 \%$ & 46 & $38,3 \%$ & 3 & $2,5 \%$ \\
\hline $\begin{array}{l}\text { 2. Cuentas con algún familiar que te } \\
\text { brinde apoyo en tus tareas escolares }\end{array}$ & 20 & $16,7 \%$ & 47 & $39,2 \%$ & 51 & $42,5 \%$ & 2 & $1,7 \%$ \\
\hline $\begin{array}{l}\text { 3. Tu familia se preocupa por el } \\
\text { avance de tus estudios }\end{array}$ & 2 & $1,7 \%$ & 85 & $70,8 \%$ & 27 & $22,5 \%$ & 6 & $5,0 \%$ \\
\hline $\begin{array}{l}\text { 4. Tus padres te dicen que puedes } \\
\text { avanzar hasta terminar el nivel de } \\
\text { educación secundaria }\end{array}$ & 3 & $2,5 \%$ & 113 & $94,2 \%$ & 1 & $0,8 \%$ & 3 & $2,5 \%$ \\
\hline $\begin{array}{l}\text { 5. Tu familia cuenta con los medios } \\
\text { económicos para apoyarte en los } \\
\text { estudios }\end{array}$ & 16 & $13,3 \%$ & 63 & $52,5 \%$ & 39 & $32,5 \%$ & 2 & $1,7 \%$ \\
\hline $\begin{array}{l}\text { 6. Tus hermanos se preocuparon por } \\
\text { continuar con estudios superiores }\end{array}$ & 64 & $53,3 \%$ & 45 & $37,5 \%$ & 8 & $6,7 \%$ & 3 & $2,5 \%$ \\
\hline $\begin{array}{l}\text { 7. Tus padres se preocuparon por } \\
\text { terminar sus estudios secundarios }\end{array}$ & 29 & $24,2 \%$ & 55 & $45,8 \%$ & 33 & $27,5 \%$ & 3 & $2,5 \%$ \\
\hline $\begin{array}{l}\text { 8. Cuentas en casa con los ambientes } \\
\text { adecuados para desarrollar tus } \\
\text { estudios }\end{array}$ & 7 & $5,8 \%$ & 55 & $45,8 \%$ & 52 & $43,3 \%$ & 6 & $5,0 \%$ \\
\hline
\end{tabular}

Nota: Instrumento de evaluación

La tabla 2, se evidencia los factores familiares que afectan el rendimiento académico de los estudiantes de la tropa evaluada, se aprecia que el 49,2\% de los evaluados consideran que su familia no los apoya, agregando que su familia no se preocupa por el avance de sus estudios. Respecto a la situación económica familiar, el 52,5\% manifiesta que sus familias no cuentan con los recursos suficientes para continuar con los estudios. Esto permite indicar que las familias presentan dificultades para convertirse en un medio negativo que afecta el rendimiento académico del grupo estudiado.

Tabla 3. Factor docente que afectan el rendimiento académico de los estudiantes de la tropa.

\begin{tabular}{lcccccccc}
\hline & \multicolumn{2}{c}{ Nunca } & \multicolumn{2}{c}{ Casi nunca } & \multicolumn{2}{c}{ Casi siempre } & \multicolumn{2}{c}{ Siempre } \\
\cline { 2 - 8 } & fi & $\%$ & fi & $\%$ & fi & $\%$ & fi & $\%$ \\
\hline $\begin{array}{l}\text { 1. Los docentes asisten con normalidad } \\
\text { a dictar las clases }\end{array}$ & 22 & $18,3 \%$ & 74 & $61,7 \%$ & 22 & $18,3 \%$ & 2 & $1,7 \%$ \\
$\begin{array}{l}\text { 2. Los docentes son puntuales con el } \\
\text { inicio de las clases }\end{array}$ & 7 & $5,8 \%$ & 75 & $62,5 \%$ & 38 & $31,7 \%$ & 0 & $0,0 \%$ \\
$\begin{array}{l}\text { 3. Los docentes brindan oportunidades } \\
\text { a todos los estudiantes para aprender } \\
\begin{array}{l}\text { 4. Los docentes brindan buen trato en } \\
\text { las clases a todos los estudiantes }\end{array}\end{array}$ & 14 & $11,7 \%$ & 66 & $55,0 \%$ & 40 & $33,3 \%$ & 0 & $0,0 \%$ \\
\hline
\end{tabular}

Nota: Instrumento de evaluación 
En cuanto a los factores docente, se observa en la Tabla 3, la puntuación más se obtuvo en el criterio trato, el 72,5\% considera que el docente no le brinda un buen trato en clase, seguido de una impuntualidad en el inicio de actividades, acompañado de inasistencias a las clases y falta de oportunidades a los estudiantes en las tareas. En resumen, los docentes no permiten un mejor rendimiento académico, más bien lo afectan convirtiéndose en un factor negativo para el logro de los aprendizajes del grupo evaluado.

Tabla 4. Factor escuela como factor que afectan el rendimiento académico de los estudiantes de la tropa.

\begin{tabular}{|c|c|c|c|c|c|c|c|c|}
\hline & \multicolumn{2}{|c|}{ Nunca } & \multicolumn{2}{|c|}{ Casi nunca } & \multicolumn{2}{|c|}{ Casi siempre } & \multicolumn{2}{|c|}{ Siempre } \\
\hline & fi & $\%$ & fi & $\%$ & fi & $\%$ & fi & $\%$ \\
\hline $\begin{array}{l}\text { 1. Las clases se brindan por medios a } \\
\text { los cuales puedes acceder }\end{array}$ & 18 & $15,0 \%$ & 67 & $55,8 \%$ & 33 & $27,5 \%$ & 2 & $1,7 \%$ \\
\hline $\begin{array}{l}\text { 2. La escuela te brinda los materiales } \\
\text { necesarios para desarrollar tus tareas }\end{array}$ & 14 & $11,7 \%$ & 75 & $62,5 \%$ & 31 & $25,8 \%$ & 0 & $0,0 \%$ \\
\hline $\begin{array}{l}\text { 3. Los profesores con que cuenta la } \\
\text { escuela están calificados para enseñar }\end{array}$ & 2 & $1,7 \%$ & 73 & $60,8 \%$ & 44 & $36,7 \%$ & 1 & $0,8 \%$ \\
\hline $\begin{array}{l}\text { 4. La escuela se preocupa por el } \\
\text { avance de tus estudios }\end{array}$ & 6 & $5,0 \%$ & 84 & $70,0 \%$ & 29 & $24,2 \%$ & 1 & $0,8 \%$ \\
\hline $\begin{array}{l}\text { 5. La escuela se comunica contigo en } \\
\text { caso dejas de asistir a clases }\end{array}$ & 5 & $4,2 \%$ & 84 & $70,0 \%$ & 29 & $24,2 \%$ & 2 & $1,7 \%$ \\
\hline
\end{tabular}

Nota: Instrumento de evaluación

El factor escuela, también se convierte en un aspecto importante que afecta el rendimiento académico del grupo evaluados, resaltando que existe poca preocupación por parte de la escuela en los avances académicos, no hay interés por la ausencia del estudiante a clases, falta de medios para acceder a clases y no se brinda materiales necesarios para el cumplimiento con las tareas académicas.

Tabla 5.Factor motivación y afecto en el rendimiento académico de los estudiantes de la tropa.

\begin{tabular}{|c|c|c|c|c|c|c|c|c|}
\hline & \multicolumn{2}{|c|}{ Nunca } & \multicolumn{2}{|c|}{ Casi nunca } & \multicolumn{2}{|c|}{ Casi siempre } & \multicolumn{2}{|c|}{ Siempre } \\
\hline & fi & $\%$ & fi & $\%$ & $\mathrm{fi}$ & $\%$ & fi & $\%$ \\
\hline $\begin{array}{l}\text { 1. Los docentes te motivan a seguir } \\
\text { avanzando en tus estudios }\end{array}$ & 20 & $16,7 \%$ & 62 & $51,7 \%$ & 35 & $29,2 \%$ & 3 & $2,5 \%$ \\
\hline $\begin{array}{l}\text { 2. Los docentes valoran tu esfuerzo } \\
\text { que realizas en las clases }\end{array}$ & 3 & $2,5 \%$ & 80 & $66,7 \%$ & 35 & $29,2 \%$ & 2 & $1,7 \%$ \\
\hline $\begin{array}{l}\text { 3. Los docentes te brindan seguridad } \\
\text { para realizar tus tareas }\end{array}$ & 11 & $9,2 \%$ & 71 & $59,2 \%$ & 37 & $30,8 \%$ & 1 & $0,8 \%$ \\
\hline $\begin{array}{l}\text { 4. Te llevas bien con tus amigos de } \\
\text { clase }\end{array}$ & 9 & $7,5 \%$ & 90 & $75,0 \%$ & 18 & $15,0 \%$ & 3 & $2,5 \%$ \\
\hline 5. Tus amigos respetan tus derechos & 17 & $14,2 \%$ & 81 & $67,5 \%$ & 18 & $15,0 \%$ & 4 & $3,3 \%$ \\
\hline $\begin{array}{l}\text { 6. Realizas tareas con tus compañeros } \\
\text { de clase }\end{array}$ & 18 & $15,0 \%$ & 68 & $56,7 \%$ & 33 & $27,5 \%$ & 1 & $0,8 \%$ \\
\hline $\begin{array}{l}\text { 7. Las relaciones interpersonales con } \\
\text { tus compañeros son satisfactorias }\end{array}$ & 13 & $10,8 \%$ & 57 & $47,5 \%$ & 47 & $39,2 \%$ & 3 & $2,5 \%$ \\
\hline
\end{tabular}

Nota: Instrumento de evaluación

Otro de los factores importantes es la motivación y afecto del estudiante, el cual juega un rol importante dentro del individuo, en el estudio es preocupante observar que los estudiantes no motivan a 
seguir avanzando académicamente, poca participación en trabajos con los compañeros, además se resalta las escasas relaciones interpersonales lo que afecta de alguna manera al rendimiento académico.

Tabla 6. Factor Regimiento afecta el rendimiento académico de los estudiantes de la tropa.

\begin{tabular}{lllllllll}
\hline & \multicolumn{2}{c}{ Nunca } & \multicolumn{2}{c}{ Casi nunca } & \multicolumn{2}{c}{ Casi siempre } & \multicolumn{2}{c}{ Siempre } \\
\cline { 2 - 8 } & fi & $\%$ & fi & $\%$ & fi & $\%$ & fi & $\%$ \\
\hline $\begin{array}{l}\text { 1. En el Regimiento te dan facilidades } \\
\text { para realizar tus estudios }\end{array}$ & 13 & $10,8 \%$ & 53 & $44,2 \%$ & 53 & $44,2 \%$ & 1 & $0,8 \%$ \\
$\begin{array}{l}\text { 2. En el regimiento te brindan el } \\
\text { tiempo necesario para tus estudios }\end{array}$ & 3 & $2,5 \%$ & 104 & $86,7 \%$ & 13 & $10,8 \%$ & 0 & $0,0 \%$ \\
$\begin{array}{l}\text { 3. En el regimiento tus superiores te } \\
\text { motivan a seguir tus estudios }\end{array}$ & 7 & $5,8 \%$ & 65 & $54,2 \%$ & 47 & $39,2 \%$ & 1 & $0,8 \%$ \\
$\begin{array}{l}\text { 4. En el Regimiento tienes los } \\
\text { espacios para estudiar }\end{array}$ & 3 & $2,5 \%$ & 78 & $65,0 \%$ & 38 & $31,7 \%$ & 1 & $0,8 \%$ \\
$\begin{array}{l}\text { 5. En el Regimiento te brindan } \\
\text { materiales educativos para desarrollar } \\
\text { tus estudios }\end{array}$ & 32 & $26,7 \%$ & 54 & $45,0 \%$ & 32 & $26,7 \%$ & 2 & $1,7 \%$ \\
\hline
\end{tabular}

Nota: Instrumento de evaluación

En lo que respecta al Regimiento, Tabla 6, como factor causante de un rendimiento académico, los datos nos indican que el Regimiento no le brinda el tiempo necesario para estudiar, no se cuenta con espacios suficientes, los superiores no motivan a continuar con sus estudios y no se le facilita materiales educativos para desarrollar sus estudios. Esto demuestra que es el Regimiento no es un factor de ayuda en el rendimiento académico en la tropa evaluada.

\subsection{PROPUESTA DE MEJORA}

A la luz de los resultados, se realiza una propuesta de un conjunto de estrategias basadas en las ideas de Orellana (2020), Cartagena (2008), Calero (2005), Iglesias (2008) y Andrade (2018), estos autores señalan que la planificación del estudiante, el ambiente donde aprende y la infraestructura con que se cuenta son importantes en la enseñanza aprendizaje. La propuesta de estrategias está basada en lo que señala el Ministerio de Educación (2016) a través del Currículo Nacional de Educación Básica, ya que es este documento técnico el que brinda las orientaciones metodológicas que deben ser tomadas en cuenta por los docentes en la planificación, ejecución y evaluación de los procesos de enseñanza y aprendizaje. El objetivo general de la propuesta es: Diseñar estrategias para disminuir las causas que afectan el rendimiento académico de la tropa del Regimiento ubicado en el distrito de Suyo.

La metodología que se propone, implica desarrollar un proceso de socialización de la información que contiene la propuesta, a través de conferencias virtuales, de acuerdo a la disponibilidad que tienen los agentes educativos para acceder a través de medios virtuales, pudiendo ser éstos, Zoom; Meet y/o WhatsApp. A la vez, se describirá y explicará de forma detallada las estrategias propuestas para cada 
problemática encontrada, en razón de las causas que provocan el bajo rendimiento académico, con la finalidad que se pueda implementar y minimizar las causas que provocan el bajo rendimiento.

Tabla 7 Propuesta de estrategias para disminuir las causas que afectan el rendimiento académico.

\begin{tabular}{|c|c|}
\hline DIMENSIÓN & ELEMENTO \\
\hline \multirow{5}{*}{$\begin{array}{l}\text { Factor asociado al } \\
\text { estudiante }\end{array}$} & Nivel de responsabilidad de los estudiantes con las clases. \\
\hline & Estrategias de aprendizaje \\
\hline & Planificación del tiempo \\
\hline & Ambiente adecuado para los estudios \\
\hline & Uso de recursos para el desarrollo de las tareas \\
\hline \multirow{4}{*}{$\begin{array}{l}\text { Factor asociado a la } \\
\text { familia }\end{array}$} & Escaso apoyo familiar para el cumplimiento de las tareas \\
\hline & Poca motivación de la familia para culminar con sus estudios secundarios. \\
\hline & Escasos recursos económicos \\
\hline & Escasa valoración de la educación por parte de la familia \\
\hline \multirow{4}{*}{$\begin{array}{l}\text { Factor asociado al } \\
\text { factor docente }\end{array}$} & Irresponsabilidad docente en su asistencia y puntualidad con las clases \\
\hline & Trato inadecuado por parte del docente \\
\hline & Estrategias inadecuadas en el desarrollo de las clases en aula \\
\hline & Uso inadecuado de las tareas escolares que se asignan a los estudiantes \\
\hline \multirow{4}{*}{$\begin{array}{l}\text { Factor asociado al } \\
\text { factor escuela }\end{array}$} & Dificultades para utilizar medios de acceso a las actividades académicas. \\
\hline & $\begin{array}{l}\text { Escaso apoyo por parte de la escuela con material didáctico que apoye en las } \\
\text { tareas escolares }\end{array}$ \\
\hline & Docentes que no califican para asumir el desarrollo de clases académicas \\
\hline & $\begin{array}{l}\text { Desinterés de la escuela por monitorear la continuidad y culminación de los } \\
\text { estudios de sus estudiantes }\end{array}$ \\
\hline \multirow{3}{*}{$\begin{array}{l}\text { Factor asociado a la } \\
\text { motivación y } \\
\text { afectividad }\end{array}$} & $\begin{array}{l}\text { Escasa motivación por parte de los docentes para que los estudiantes culminen } \\
\text { con sus estudios. }\end{array}$ \\
\hline & Relaciones personales inadecuadas entre estudiantes \\
\hline & Desinterés de los estudiantes por culminar con sus estudios \\
\hline \multirow{2}{*}{$\begin{array}{l}\text { Factor asociado al } \\
\text { Regimiento }\end{array}$} & $\begin{array}{l}\text { Escaso apoyo del regimiento militar para que los soldados culminen con sus } \\
\text { estudios }\end{array}$ \\
\hline & $\begin{array}{l}\text { Condiciones inadecuadas en el regimiento militar para que los estudiantes } \\
\text { puedan estudiar }\end{array}$ \\
\hline
\end{tabular}

\section{DISCUSIÓN}

Los resultados nos muestran que, la mayoría de los soldados no asisten con normalidad a sus clases, sin justificación alguna, evidenciándose irresponsabilidad de su parte. Además, no se organizan para desarrollar sus estudios, es decir no planifican horarios y/o métodos de estudio, a ello, se suma el hecho que no cuentan con un espacio adecuado, textos y dispositivos para desarrollar sus tareas, todo esto tiene una repercusión en el rendimiento académico, resultados distintos reportó Gómez y Merino (2018), ya que concluye que los estudiantes evidencian un nivel adecuado en el uso de las estrategias de aprendizaje con respecto a la administración del tiempo y a la concentración, siendo fundamental en su aprendizaje.

Sin duda, los factores personales sino están bien fundamentados van a generar bajos rendimientos en los estudios, tal como lo señala Álvaro (1990), quien considera que los factores personales influyen en el rendimiento, enfatizando como una de las causas más significativas la inteligencia, la cual muestra 
correlación moderada y bastante variable con el rendimiento. Por su lado, Barreiro (2001) también sostiene como factores personales que promueven el fracaso académico a la falta de atención y concentración durante el desarrollo de las sesiones de aprendizaje, la falta de planificación y/o priorización de tiempo dedicado al estudio, los problemas personales que aquejan al estudiante y la priorización de mayor tiempo en actividades de ocio como: tv, reuniones con amigos, deportes.

En cuanto a los factores familiares que inciden en el rendimiento académico, más de la mitad de los evaluados señalan que, en su hogar no cuentan con una persona que les brinde apoyo escolar, además la familia no muestra interés para que estos culminen el nivel secundario, esto se relaciona con el alto porcentaje de hermanos y padres de familia que no han realizado estudios superiores y secundarios respectivamente y no cuenta con los medios suficientes para apoyarlos, el estudio incide en la formación de los padres como uno de los aspectos importante en la continuidad de los estudios de los soldados evaluados, tal como lo señala Fajardo y Maestre (2017), los autores demuestran que un nivel alto de formación académica de los padres y madres, así como pertenecer a las clases ocupacionales medias o altas, predicen el buen rendimiento académico en sus hijos.

El nivel educativo de los familiares adultos quienes están a cargo de los estudiantes, aparece como indicador relevante al igual que el aspecto socioeconómico que incide de forma negativa el nivel de calidad educativa, de la misma forma el contexto geográfico o regional en el que se forma el estudiante en época escolar (Garbanzo, 2013).

$\mathrm{Al}$ analizar los factores de la práctica docente en el aula y el rendimiento académico, los resultados nos permiten señalar que no hay una buena práctica docente caracterizado por irresponsabilidad en el cumplimiento de su labor pedagógica, además, no les brindan buen trato y las oportunidades necesarias para aprender. La situación es más grave, pues señalan que, no entienden las clases que dictan los docentes y no utilizan estrategias de trabajo en grupo, u otras estrategias que los motiven a aprender.

$\mathrm{Al}$ respecto Grade (2018) revela como uno de los factores que conllevan a resultados académicos positivos a la práctica docente, del aula y escuela, sin embargo, nos damos cuenta que en el estudio realizado es, al contrario, docentes poco comprometidos con su labor que genera desmotivación y bajos aprendizajes. Estos resultados están en concordancia con lo encontrado por Garbanzo (2013) se pone énfasis a las estrategias metodológicas que utilizan los profesores, los horarios de las diversas áreas, o las metas de estudiantes asignadas por docente como aspectos relevantes para el logro de los objetivos académicos.

El factor Escuela que inciden en el rendimiento académico, los resultados de la investigación arrojaron que la escuela no cuenta con los medios adecuados para desarrollar las clases, además no se brindan los materiales necesarios, lo que limita el aprendizaje. Por otro lado, consideran que los docentes no son competentes en su labor y no demuestran interés por el aprendizaje de los soldados. Los hallazgos 
en la presente investigación se relacionan con lo encontrado por Sánchez (2017) quien concluye que la mayor parte de los estudiantes, en promedio el $65 \%$ de ellos, considera que su rendimiento esta influenciado por los factores sociales e institucionales. En este sentido Gonzáles (2003), señal que los factores escolares, tienen incidencia en el rendimiento académico aspectos de naturaleza procesual y funcional (clima escolar) en relación a los aspectos estructurales (recursos materiales).

La falta de motivación por parte de los docentes para que los soldados continúen con sus estudios, es un aspecto que según los soldados se da de manera cotidiana. En relación a las interacciones con sus compañeros de clase, manifiestan que estas relaciones sociales no son buenas, lo cual se expresa en la falta de compañerismo para realizar tareas en equipo, todo ello conlleva a que los soldados manejen bajas expectativas para culminar sus estudios. Los resultados del estudio destacan que el factor motivación del docente y las estrategias que implemente para los aprendizajes es un factor importante en rendimiento académico, si estos dos procesos no están bien trabajados tendremos dificultades en el logro de los aprendizajes del grupo, esto concuerda con lo que señala Méndez (2016) dado que menciona que las estrategias que implementan los docentes además de la motivación que brindan a los estudiantes, influyen sobre el rendimiento que evidencian los alumnos.

Por su parte, Vásquez (2012) refiere que las expectativas, el contexto familiar y la motivación influye de alguna u otra manera en los logros académicos. Los hallazgos también coinciden con el planteamiento que señala que los factores motivacionales, las aspiraciones y las perspectivas académicas tienen una influencia en el rendimiento académico, pues, se relaciona bastante con las facultades causales y se asocia de forma positiva con el rendimiento.

En cuanto al Regimiento, que es el espacio donde más de desarrolla el estudiante soldado, en el estudio se observó que no hay facilidades del regimiento, pues no les dan el tiempo necesario para sus estudios ni son motivados para seguir sus estudios, además, señalan que el regimiento no cuenta con los espacios y materiales para estudiar. Salas (2004) sostiene que, cuando al estudiante le afectan situaciones de las cuales le es bastante difícil salir, generalmente sufre las consecuencias del fracaso escolar, entendiéndose a este como el hecho de no lograr las competencias básicas para desenvolverse de forma independiente y autónoma en la sociedad.

\section{CONCLUSIÓN}

Sobre las características personales del estudiante, se destacan la baja responsabilidad que tiene la muestra para asistir a sus clases, casi nunca planificas sus actividades académicas, bajos hábitos de estudio, carencia de ambiente y materiales que les permitan cumplir con sus actividades escolares. 
Respecto a los rasgos que caracterizan al factor familia, los evaluados no cuentan con apoyo por parte de su familia, escasos medios económicos, además, los padres y hermanos, no han continuado estudios secundarios y superiores respectivamente, lo que lo convierte en un factor importante dentro de los aprendizajes.

Sobre el factor docente y práctica de aula, los docentes muestran irresponsabilidad en la asistencia de clases, además no brindan un buen trato y han sido pocas las oportunidades que ofrecen a sus estudiantes para aprender, también señalan que los docentes no explican de forma clara las clases y las tareas que realizan no son revisadas y no reciben orientaciones al respecto.

En el caso del factor escuela, se evidencia que esta, no les brinda los materiales necesarios para desarrollar las tareas que se generan en clases, además de no mostrar preocupación por el avance de sus estudios.

En relación al factor motivacional y afectivo, los evaluados señala que no reciben motivación por parte de los docentes para continuar estudios, casi nunca se llevan bien con sus compañeros, situación que conlleva a que no se realicen tareas grupales y casi nunca muestran optimismo para culminar sus estudios.

Sobre el factor Regimiento, que casi nunca les brindan tiempo necesario para cumplir con sus estudios y no los motivan dentro del regimiento, finalmente, no existen los ambientes y materiales necesarios para estudiar.

Estos hallazgos permiten reconocer la importancia de la propuesta de estrategias para disminuir las causas que afectan el rendimiento académico en el grupo estudiado, dado que está centrada en atacar a los factores personales, familiares, docente, escuela, motivación y afecto y regimiento. 


\section{REFERENCIAS}

Adell, M. (2006). Estrategias para mejorar el rendimiento académico de los adolescentes. Madrid: Psicología Pirámide.

Álvaro Page, M. (1990) Hacia un modelo causal de rendimiento. Madrid: CIDE.

Andrade, A. (2018) Diagnóstico y plan de mejora para el uso de dispositivos electrónicos de los estudiantes de primero de secundaria de la Unidad Educativa Montepiedra. (Tesis de maestría) UDEP Piura.

Barreiro, F. (2001) Un reto en el siglo XXI: El fracaso escolar. Perspectiva del profesorado. Revista de Ciencias de la Educación, 187, 325-339.

Brengelmann, J. (1975) Personalidad y rendimiento. En V. Pelechano (Dir.), Psicología estimular y modulación. Madrid: Manova

Buelvas, E. (2018) Estrategias de aprendizaje y rendimiento académico en los estudiantes de secundaria El Carmen, (tesis doctoral) Universidad Privada Nolbert Wiener.

Calero M. (2005) Técnicas de estudio e investigación. Edit. San Marcos. Lima, 2005.

Carrizales, A. (2017) Estrategias didácticas para el rendimiento académico en los estudiantes, (tesis doctoral) Universidad César Vallejo Perú.

Cartagena, M. (2008). Relación entre la autoeficacia y el rendimiento. Revista iberoamericana sobre calidad,eficacia y cambio en educación, Volumen 6, Número 3.

Carabaña, J. (1979) Origen social, inteligencia y rendimiento académico al final de la EGB. En INCE (Ed.) Temas de investigación educativa. Madrid: Servicio de Publicaciones del MEC.

Creemers, B. y Scheerens, J. (1989) Development in school effectiveness research. International Journal of Educational Research..

Fajardo, F. y Maestre, M. (2017) Análisis del rendimiento académico de los alumnos de educación secundaria, según las variables familiares", (tesis doctoral) Universidad de Educación a Distancia de Madrid España.

Garbanzo, G. (2013) Factores asociados al rendimiento académico en estudiantes universitarios desde el nivel socioeconómico (tesis doctoral) Universidad de Costa Rica.

Garijo, R. (2016) Factores determinantes del bajo Rendimiento académico en educación secundaria en la provincia de Albacete, (tesis doctoral) Universidad de educación a Distancia España.

Gómez, F. y Merino, A. (2018), Estrategias de aprendizaje y rendimiento académico empleadas por estudiantes de liceos de Talca con alto y bajo puntaje" (Tesis doctoral) Universidad Autónoma de Chile.

Gonzáles, B. (2003) Factores determinantes del bajo rendimiento académico en educación secundaria. (tesis doctoral) Universidad Complutense de Madrid. 
Good,Th. y Brophy, J. (1986) School effects. En M.C. Wittrock (Ed.) Handbook of research on teaching. Nueva York: Macmillan

Grade (2018) Factores asociados al logro académico. Tercer estudio regional comparativo y explicativo. Oficina Regional de Educación para América Latina y el Caribe.

Iglesias, M. L. (2008). Observación y Evaluación del Ambiente de Aprendizaje en Educación Infantil: Dimensiones y Variables a Considerar. Revista Iberoamericana de Educación, (47), 49-70. Recuperado de http://dialnet.unirioja.es/servlet/articulo?codigo=2736756 lnhttp://www.rieoei.o $\mathrm{rg} / \mathrm{rie} 47 \mathrm{a} 03 . \mathrm{htm}$

INCIE (1976) Determinantes del rendimiento académico. Madrid: Servicio de Publicaciones del MEC.

Lamas, H. (2015) Sobre el rendimiento escolar. Academia Peruana de Psicología, Lima, Perú.

López, M. (2017) Estrategia para la prevención del riesgo de deserción en los estudiantes en condiciones de pobreza, (tesis doctoral) Universidad Nacional Mayor de San Marcos Perú.

Marchesi, A. (2003) El fracaso escolar en España. Documento de trabajo del Laboratorio 11/2003. Fundación Alternativas. Documento electrónico

Marsh, H.W. (1984) Relations among dimensions of self-atribution, dimensions of self-concept, and academic achievements. Journal of Educational Psychology, 76, 1291-1308.

Méndez, C. (2016) Estrategias de aprendizaje y motivación en el rendimiento académico de los estudiantes, (tesis doctoral) Universidad César Vallejo, Perú.

Ministerio de educación (2019) Resolución Viceministerial № 053-2019 MINEDU. San Borga Lima Perú.

Ministerio de educación (2016) Currículo Nacional de la Educación Básica. San Borga Lima Perú.

Orellana, A. (2020) Disciplina en el aula promoviendo la responsabilidad. Recuperado https://blog.bosquedefantasias.com/noticias/disciplina-aula-promoviendo-responsabilidad

Pedraza, O. (2016) Programa de estrategias didácticas cognitivas para desarrollar la competencia comunicativa en los estudiantes de educación secundaria, (tesis doctoral) Universidad César Vallejo, Perú.

Pelechano, V. (1989) Informe del proyecto de investigación de la EGB y BUP. Análisis y modificación de Conducta, 15. Número Monográfico

Purkey, S.C. y Smith, M.S. (1985) Educational policy and school effectiveness. En G. Austin y M. Garber (Eds.) Research on exemplary schools. Nueva York: Academic Press.

Rodríguez, I. y Madrigal, A. (2016) Rendimiento académico y estrategias de aprendizaje. Universidad Autónoma del Estado de México,

Rodríguez, S. (1982) Un modelo de predicción del rendimiento académico en la $2^{\text {a }}$ etapa de EGB. Modelos de Investigación Educativa. Barcelona: Edicions Universitat. pp. 131-170. 
Salas, M. (2004) El fracaso escolar: estado de la cuestión. Estudio documental sobre el fracaso escolar y sus causas. $1^{\circ}$ Congreso Anual sobre fracaso escolar en Palma de Mallorca.

Sánchez, L. (2017) Factores que inciden en el rendimiento académico de los estudiantes de Ingeniería Industrial, (tesis doctoral) Universidad César Vallejo Perú.

Song, I.G. y Hattie, J. (1984) Home environment, self-concept and academic achievement: a causal modeling approach. Journal of Educational Psychology, 76, 1269-1281.

Vásquez, C. (2012) Factores de impacto en el rendimiento académico universitario. Un estudio a partir de las percepciones de los estudiantes. Universidad Nacional de Rosario.

Velasco, G. (2016) El rendimiento académico en el nivel de educación secundaria en Chiapas México, (tesis doctoral) Universidad Autónoma de Barcelona. 